\title{
Intoxicação experimental por Trema micrantha (Ulmaceae) em caprinos ${ }^{1}$
}

\author{
Sandra Davi Traverso ${ }^{2}$, André Mendes Ribeiro Corrêa ${ }^{2}$, Caroline Argenta Pescador ${ }^{2}$, \\ Edson Moleta Colodel ${ }^{3}$, Cláudio Estêvão Farias da $\mathrm{Cruz}^{2}$ e David Driemeier ${ }^{2}$
}

\begin{abstract}
Traverso S.D., Corrêa A.M.R., Pescador C.A., Colodel E.M., Cruz C.E.F. \& Driemeier D. 2002. [Experimental poisoning by Trema micrantha (Ulmaceae) in goats.] Intoxicação experimental por Trema micrantha (Ulmaceae) em caprinos. Pesquisa Veterinária Brasileira 22(4):141-147. Setor de Patologia Veterinária, Depto Patologia Clínica Veterinária, Faculdade de Veterinária, Universidade Federal do Rio Grande do Sul, Av. Bento Gonçalves 9090, Cx. Postal 15094, Porto Alegre, RS 91540-000, Brazil. E-mail: davetpat@vortex.ufrgs.br

Green leaves of Trema micrantha were ground and mixed with water in a domestic blender and then administered by stomach tube to seven goats. One additional goat was fed ad libidum with the green leaves of T. micrantha. Clinical signs were observed in six goats that became ill 2 days after having been dosed with or fed the plant. There were five deaths, which occurred until 4 days after ingestion. Affected goats remained static during long periods and kept their heads low. Incoordination, rhythmical movements of the head, apathy, anorexia, and tenesmus were also noticed. Paddling movements and coma were seen in one goat. T. micrantha was toxic at dosages of $30 \mathrm{~g} / \mathrm{kg}$ or higher. The most significant gross lesions were observed in the livers, which were yellowish, friable, and with pronounced lobular pattern. Their cut surfaces were reddened and depressed areas alternated with whitish ones. The liver of one goat was slightly but homogeneously reddened but did not show accentuated lobulation. Petechial haemorrhages in the region between the chest and scapula, in the epicardium, mediastinum and serosal membranes of the abdominal organs were also observed. The most important histologic finding was hepatic centrilobular coagulative necrosis, which was associated with congestion, haemorrhages and degenerative changes in the circumjacent hepatocytes. Additional microscopic lesions were found in the nervous system and included perineuronal and perivascular edema and swollen neurones, especially those of the frontal cortex.
\end{abstract}

INDEX TERMS: Poisonous plants, plant poisoning, Trema micrantha, Ulmaceae, goats.

RESUMO.- Sete caprinos receberam, por sonda esofágica, uma suspensão aquosa de folhas verdes de Trema micrantha moídas e um outro recebeu folhas verdes da planta à vontade. Os sinais clínicos iniciaram-se 2 dias após a ingestão e ocorreram em seis caprinos, cinco dos quais morreram em até 4 dias. Os animais doentes permaneciam longos períodos em estação, com cabeça baixa, olhar fixo, apáticos e inapetentes.

\footnotetext{
${ }^{1}$ Aceito para publicação em 30 de agosto de 2002.

${ }^{2}$ Depto Patologia Clínica Veterinária, UFRGS, Cx. Postal 15094, Porto Alegre, RS 91540-000. Autor para correspondência. E-mail: davetpat@vortex.ufrgs.br

${ }^{3}$ Depto Clínica Médica Veterinária, Universidade Federal de Mato Grosso (UFMT), Cuiabá, MT.
}

Tenesmo, incoordenação e movimentos rítmicos laterais da cabeça também foram observados. T. micrantha mostrou-se tóxica a partir de $30 \mathrm{~g} / \mathrm{kg}$ de peso corporal. A alteração macroscópica mais significativa foi observada no fígado, que se apresentou friável, amarelado e com acentuado padrão lobular. Ao corte, havia áreas vermelhas, deprimidas e entremeadas por áreas mais claras. Em um animal, a coloração do fígado era vermelha, homogênea, mais clara que o normal e sem evidenciação do padrão lobular. Petéquias foram constatadas entre a escápula e o esterno, no epicárdio, no mediastino e nas serosas dos órgãos da cavidade abdominal. A principal alteração histológica foi necrose coagulativa centrolobular que, em alguns casos, atingia todo o lóbulo, associada à congestão, hemorragia e alterações degenerativas nos hepatócitos circunjacentes. No sistema nervoso, havia tume- 
fação de neurônios, mais proeminente no córtex frontal, associado a edema perineuronal e perivascular.

TERMOS DE INDEXAÇÃO: Plantas tóxicas, intoxicação por plantas, Trema micrantha, Ulmaceae, caprinos.

Classificação CNPq: 5.05.03.00-6 Patologia Animal.

\section{INTRODUÇÃO}

Trema micrantha ("Grandiúva") é uma espécie arbórea nativa do Brasil, de crescimento rápido, copa densa e intolerante à sombra, características estas que explicam sua freqüente utilização como pioneira em sistemas de reflorestamento e recuperação de solos degradados (Nowotny \& Nowotny 1993, Castellani \& Aguiar 1998). Suas folhas são recomendadas para tratamento de doenças da pele, sífilis e reumatismo (Lorenzi 2000) e seus extratos têm demonstrado atividades analgésicas e antiinflamatórias em ratos e camundongos (Barbera et al. 1992). Durante períodos de estiagem, as folhas de $T$. micrantha servem de alimento para o gado (Lorenzi 2000).

No Rio Grande do Sul, a intoxicação espontânea por $T$. micrantha em cabras, foi previamente descrita (Traverso et al. 2002), em novo surto diagnosticado pelos autores. T. micrantha causou a morte de todas as sete cabras que pastoreavam em um piquete, onde uma árvore foi derrubada.

Há suspeitas de casos de intoxicação por T. micrantha em bovinos no Estado de Santa Catarina, onde forragens obtidas daquela planta são usualmente fornecidas para essa espécie (Gava 2000, comunicação pessoal). T. micrantha foi também descrita como tóxica, experimentalmente, em coelhos que a ingeriram espontaneamente em doses a partir de $35 \mathrm{~g} / \mathrm{kg}$ de peso vivo (Traverso \& Driemeier 2000). Casos de intoxicação natural causados por outra espécie do gênero, Trema tomentosa, foram descritos em bovinos, caprinos, ovinos (Mulhearn 1942), equiinos (Hill et al. 1985), camelos (Trueman \& Powell 1991) e veados (McKenzie 1985). Experimentalmente, T. tomentosa foi demonstrada como tóxica para bovinos e caprinos nas doses de $344 \mathrm{~g}$ e $1140 \mathrm{~g}$, respectivamente (Mulhearn 1942), e seus extratos, contendo o princípio ativo denominado trematoxina, provocaram lesões hepatotóxicas em roedores (Oelrichs 1968).

O quadro clínico-patológico observado nas intoxicações naturais por T. micrantha tem características em comum com várias hepatotoxicoses agudas causadas por plantas. Relatos de hepatotoxicoses agudas com ocorrência no Brasil têm incluído Cestrum laevigatum em bovinos (Döbereiner et al. 1969) e caprinos (Peixoto et al. 2000), Cestrum parqui em bovinos (Riet-Correa et al. 1986), Cestrum corymbosum var. birsutum em bovinos (Gava et al. 1991), Cestrum intermedium em bovinos (Gava et al. 1996), Sessea brasiliensis em bovinos (Canella et al. 1968), Vernonia mollissima em bovinos (Döbereiner et al. 1976), Vernonia rubricaulis em bovinos (Tokarnia \& Döbereiner 1982) e Xanthium spp em bovinos (Méndez et al. 1998, Driemeier et al. 1999, Colodel et al. 2000).

O presente estudo apresenta como objetivos a caracterização do quadro clínico-patológico da intoxicação por $T$. micrantha, em caprinos, a determinação de aspectos diferenciais em relação às demais hepatopatias tóxicas agudas e 0 estabelecimento da dose tóxica.

\section{MATERIAL E MÉTODOS}

Trema micrantha (Fig. 1 e 2) foi coletada no Município de Porto Alegre/ RS. Foram utilizados nove caprinos de ambos os sexos, com idade aproximada de 4 meses. Estes animais foram numerados de 1 a 9 (Quadro 1). Os Caprinos 1, 2, 3, 4, 5, 7 e 8 receberam em dose única, por sonda esofágica, uma suspensão aquosa preparada em liqüidificador doméstico, contendo folhas verdes de T. micrantha. As doses administradas aos animais foram de $35 \mathrm{~g} / \mathrm{kg}$ de peso corporal para os Caprinos 1 e 3, 30g/ $/ \mathrm{kg}$ para os Caprinos 2 e 4, 25g/kg para o Caprino 5 e $40 \mathrm{~g} / \mathrm{kg}$ para os Caprinos 7 e 8 . O Caprino 6 recebeu folhas verdes da planta à vontade, mas somente durante um dia, comendo $35,4 \mathrm{~g} / \mathrm{kg}$ da planta, enquanto que o Caprino 9 foi usado como controle. Estes caprinos foram mantidos em baias de alvenaria e alimentados com feno de alfafa e ração comercial.

Coletas de sangue foram realizadas em intervalos de 24 horas para dosagem de gama-glutamil transferase (GGT), bilirrubina direta e bilirrubina indireta. Dosagens adicionais de glicose foram realizadas no Caprino 8. Uma coleta de sangue prévia à administração da planta serviu como controle para cada animal. Os caprinos foram observados diariamente. Foram feitas biópsias hepáticas pelo método de punção trans-torácica, usando agulha de Menghini (Braga et al. 1985), aos 10 dias e aos 6 meses após a administração. Os caprinos que não morreram, bem como o controle, foram eutanasiados, 6 meses após a administração da planta.

Os animais foram necropsiados imediatamente após a morte e

Quadro 1. Intoxicação experimental por Trema micrantha em caprinos. Doses administradas e evolução clínica

\begin{tabular}{cccccc}
\hline Caprino & $\begin{array}{c}\text { Peso } \\
(\mathrm{kg})\end{array}$ & $\begin{array}{c}\text { Dose } \\
(\mathrm{g} / \mathrm{kg})\end{array}$ & $\begin{array}{c}\text { Quantidade } \\
(\mathrm{g})\end{array}$ & $\begin{array}{c}\text { Tempo decorrido entre a } \\
\text { administração da planta } \\
\text { e o aparecimento } \\
\text { dos sinais clínicos }\end{array}$ & $\begin{array}{c}\text { Tempo decorrido entre a } \\
\text { administração da planta } \\
\text { e a morte do animal }\end{array}$ \\
\hline 1 & 18,6 & 35 & 615 & $50 \mathrm{~h} 00 \mathrm{~min}$ & $72 \mathrm{~h} 00 \mathrm{~min}$ \\
2 & 23,0 & 30 & 690 & $48 \mathrm{~h} 00 \mathrm{~min}$ & $58 \mathrm{~h} 30 \mathrm{~min}$ \\
3 & 17,2 & 35 & 602 & $48 \mathrm{~h} 00 \mathrm{~min}$ & $51 \mathrm{~h} 00 \mathrm{~min}$ \\
4 & 17,4 & 30 & 522 & $49 \mathrm{~h} 00 \mathrm{~min}$ & - \\
5 & 13,4 & 25 & 585 & Não adoeceu & $77 \mathrm{~h} 00 \mathrm{~min}$ \\
6 & 21,2 & 35,4 & 750 & Não adoeceu & - \\
7 & 29,0 & 40 & 1140 & 48 e $102 \mathrm{~h} 00 \mathrm{~min}$ & $108 \mathrm{~h} 00 \mathrm{~min}$ \\
8 & 8.6 & 40 & 360 & Não adoeceu & -
\end{tabular}


fragmentos de todos os órgãos, incluindo o encéfalo e medula espinhal, foram coletados e fixados em formalina tamponada a $10 \%$. As amostras foram processadas rotineiramente para exame histológico e corados pela técnica de hematoxilina e eosina (HE) (Prophet 1992). Para cada encéfalo de animal experimental foi utilizado um encéfalo controle obtido de caprinos de abate e processados histologicamente de forma similar.

\section{RESULTADOS}

A evolução clínica observada na intoxicação experimental por Trema micrantha está apresentada no Quadro 1 . Sinais clínicos da intoxicação com evolução para morte foram observados em 5 animais (Caprinos 1, 3, 4, 6 e 8). Os animais que receberam $35 \mathrm{~g} / \mathrm{kg}$ (Caprinos 1 e 3), um dos dois que receberam $30 \mathrm{~g} / \mathrm{kg}$ (Caprino 4) e o que comeu planta à vontade (Caprino 6) adoeceram e morreram dentro de um período de 3 dias. 0 Caprino 8 , um dos dois que receberam $40 \mathrm{~g} / \mathrm{kg}$, morreu em 4 dias. Os primeiros sinais clínicos manifestaram-se a partir do segundo dia após a administração da planta.

\section{Sinais clínicos}

Sinais clínicos foram observados nos Caprinos 1, 2, 3, 4, 6 e 8. Destes, apenas o Caprino 2 não morreu. Nos Caprinos 1 , 3,4 e 6 , as primeiras alterações clínicas foram observadas entre 48 e 77 horas após a administração da suspensão da planta e a evolução clínica foi de 2 a 34 horas. Os animais permaneciam longos períodos em estação, relutantes à movimentação, mantendo-se com cabeça baixa e olhar fixo, apáticos, inapetentes e com tenesmo. Às vezes, apresentavam incoordenação e movimentos rítmicos laterais da cabeça. 0 Caprino 1, além dos sinais descritos, demonstrou incapacidade para beber água, balidos freqüentes, movimentos de pedalagem e, finalmente, coma durante os 10 minutos que antecederam sua morte. 0 Caprino 6 , que comeu a planta à vontade, manifestou ranger de dentes, atonia ruminal, timpanismo, balidos quando movimentado, pressão e roçar da cabeça contra a parede, deitar e levantar freqüentemente; observaram-se movimentos rítmicos dorso-ventrais da cabeça, quando em decúbito esternal.

O Caprino 8 manifestou timpanismo e apatia 48 horas após a administração da planta, 4 horas após melhora significativa, inclusive tendo voltado a se alimentar, porém com timpanismo intermitente e, no $4^{\circ}$ dia pós-administração, voltou a adoecer mostrando intensa apatia, decúbito e morte 5 horas após o reinício dos sinais clínicos mais acentuados.

O Caprino 2 apresentou apatia e diarréia no segundo dia pós-administração, mas recuperou-se rapidamente e não teve recaídas até o final do experimento, quando então foi eutanasiado. Os Caprinos 5, 7 e o controle (Caprino 9) não manifestaram quaisquer sinais clínicos.

\section{Achados laboratoriais}

Todos caprinos que morreram, bem como os Caprinos $2 \mathrm{e}$ 7 (que adoeceram, mas não morreram) apresentaram aumento da GGT. O Caprino 8 que recebeu $40 \mathrm{~g} / \mathrm{kg}$ e morreu e os Caprinos 1 e 3 que receberam $35 \mathrm{~g} / \mathrm{kg}$ tiveram aumento de bilirrubina livre. Somente o Caprino 1 teve aumento de
Quadro 2. Intoxicação experimental por Trema micrantha em caprinos. Atividade sérica de gamaglutamil transferase (GGT) e níveis séricos de bilirrubina

\begin{tabular}{|c|c|c|c|c|c|}
\hline $\begin{array}{l}\text { Caprino e do- } \\
\text { se tóxica em } \\
\mathrm{g} / \mathrm{kg} \text { de peso }\end{array}$ & $\begin{array}{l}\text { Dia da } \\
\text { coleta }^{\mathrm{a}}\end{array}$ & $\begin{array}{c}\text { GGT UI/l } \\
(6-28)\end{array}$ & $\begin{array}{c}\text { Bilirrubina } \\
\text { total } \mathrm{mg} / \mathrm{dl} \\
(0.1-1.2)\end{array}$ & $\begin{array}{c}\text { Bilirrubina } \\
\text { livre } \mathrm{mg} / \mathrm{dl} \\
(0.1-1.0)\end{array}$ & $\begin{array}{l}\text { Bilirrubina con- } \\
\text { jugada } \mathrm{mg} / \mathrm{dl} \\
(<0.4)\end{array}$ \\
\hline 1 & 10 & 29 & 0.80 & 0.70 & 0,10 \\
\hline \multirow[t]{3}{*}{$35 \mathrm{~g} / \mathrm{kg}$} & $2^{\circ}$ & 47 & 0.60 & 0.50 & 0,10 \\
\hline & $3^{\circ}$ & 64 & 0.70 & 0.60 & 0,10 \\
\hline & $4^{\circ}$ & 328 & 3.30 & 2.50 & 0,80 \\
\hline 2 & 1 은 & 58 & 0.50 & 0.40 & 0,10 \\
\hline \multirow[t]{6}{*}{$30 \mathrm{~g} / \mathrm{kg}$} & $2^{\circ}$ & 50 & 0.60 & 0.50 & 0,10 \\
\hline & $3^{\circ}$ & 52 & 0.80 & 0.70 & 0,10 \\
\hline & 40 & 64 & 0.80 & 0.70 & 0,10 \\
\hline & 5 은 & 96 & 0.30 & 0.20 & 0,10 \\
\hline & 60 & 105 & 0.80 & 0.70 & 0,10 \\
\hline & 7 은 & 120 & 0.50 & 0.40 & 0,10 \\
\hline 3 & 1 은 & 31 & 0.40 & 0.30 & 0,10 \\
\hline \multirow{2}{*}{$35 \mathrm{~g} / \mathrm{kg}$} & $2^{\circ}$ & 33 & 0.60 & 0.50 & 0,10 \\
\hline & $3^{\circ}$ & 264 & 2.40 & 2.10 & 0,30 \\
\hline 4 & 1 은 & 38 & 0.3 & 0.2 & 0,1 \\
\hline \multirow[t]{2}{*}{$30 \mathrm{~g} / \mathrm{kg}$} & $2^{\circ}$ & 39 & 0.60 & 0.50 & 0,10 \\
\hline & $3^{\circ}$ & 148 & 1.0 & 0.90 & 0,10 \\
\hline 5 & 10 & & 2.2 & 1.20 & 1,0 \\
\hline \multirow{3}{*}{$25 \mathrm{~g} / \mathrm{kg}$} & $2^{\circ}$ & 38 & 0.1 & $<0.01$ & $<0,01$ \\
\hline & 30 & 88 & 0.20 & $<0.01$ & 0,05 \\
\hline & $4^{\circ}$ & 96 & 1.38 & 1.28 & 0,10 \\
\hline 6 & 1 은 & 56 & 0.1 & $<0.01$ & $<0,01$ \\
\hline \multirow[t]{4}{*}{ A vontade } & $2^{\circ}$ & 20 & 0.1 & $<0.01$ & $<0,01$ \\
\hline & 3 은 & 74 & 0.23 & 0.20 & 0,003 \\
\hline & 4 은 & 119 & 0.77 & 0.50 & 0,027 \\
\hline & $5^{\circ}$ & 184 & 0.20 & 0.18 & 0.02 \\
\hline 7 & 10 & 38 & 0.35 & 0.25 & 0,1 \\
\hline \multirow[t]{6}{*}{$40 \mathrm{~g} / \mathrm{kg}$} & $2^{\underline{o}}$ & 51 & 0.05 & 0.04 & 0,01 \\
\hline & $3^{\circ}$ & 8 & 0.10 & $<0.01$ & $<0,01$ \\
\hline & 40 & 18 & $<0.1$ & $<0.01$ & 0,1 \\
\hline & 5 은 & 71 & 0.51 & 0.40 & 0,11 \\
\hline & 60 & 104 & 0.10 & $<$ & $<$ \\
\hline & 7 은 & 62 & 0.54 & 0.09 & 0,45 \\
\hline 8 & 1 은 & 72 & 0.20 & 0.17 & 0,03 \\
\hline \multirow[t]{5}{*}{$40 \mathrm{~g} / \mathrm{kg}$} & $2^{\circ}$ & 85 & 1.15 & 0.98 & 0,17 \\
\hline & 3 은 & 48 & 0.54 & 0.24 & 0,30 \\
\hline & 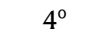 & 94 & 1.24 & 0.66 & 0,58 \\
\hline & $5^{\circ}$ & 203 & 1.38 & 0.74 & 0,64 \\
\hline & 6 은 & 181 & 2.08 & 1.78 & 0,30 \\
\hline
\end{tabular}

${ }^{\mathrm{a}} \mathrm{A}$ primeira coleta de sangue realizada antes da administração da planta aos caprinos foi usada como controle para GGT e bilirrubinas.

bilirrubina conjugada. As atividades séricas da GGT e de bilirrubina estão apresentados no Quadro 2. O Caprino 8 apresentou acentuada queda da glicemia $(110 \mathrm{mg} / \mathrm{dl}$ antes do experimento para menos de $20 \mathrm{mg} / \mathrm{dl}$ pouco antes da morte).

\section{Achados de necropsia}

A alteração mais significativa na intoxicação experimental por T. micrantha foi observada no fígado, que se apresen- 


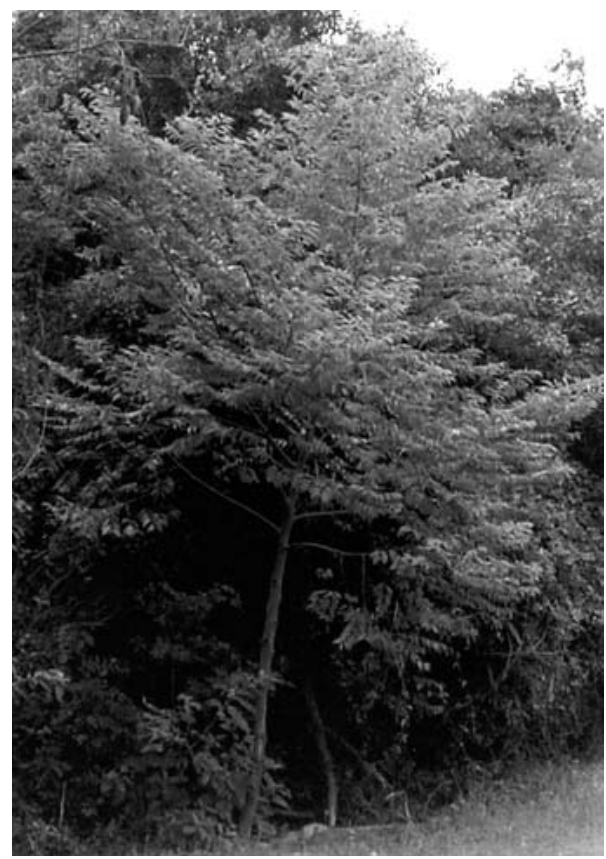

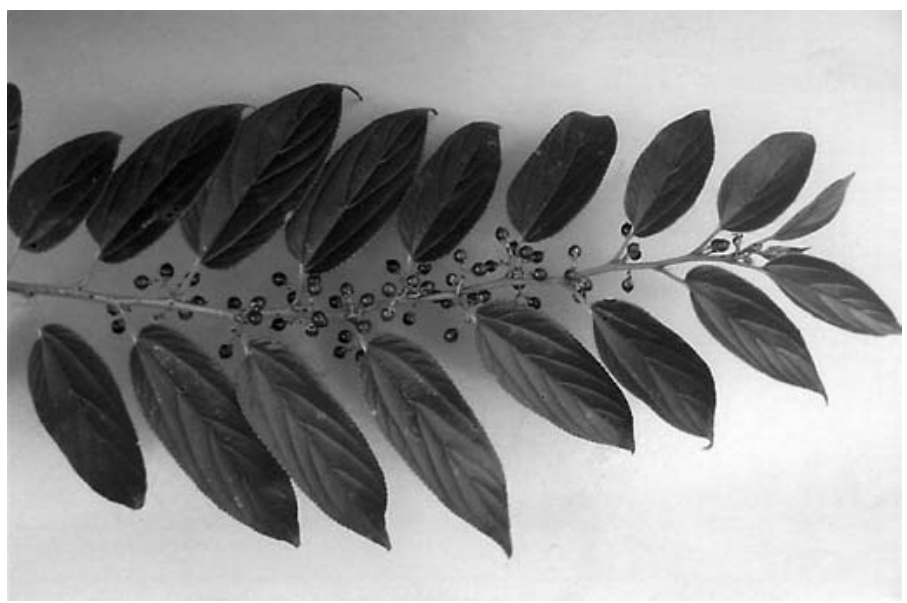

Fig. 1. Árvore de Trema micrantha de porte médio em crescimento. Maio de 2002

Fig. 2. Ramo de Trema micrantha em floração e frutificação. Fevereiro de 2001. tou friável, amarelado, com padrão lobular acentuado por áreas multifocais de hemorragias nas superfícies capsular (Fig. 3) e de corte; adicionalmente, ao corte, eram observadas áreas vermelhas, deprimidas e entremeadas por áreas mais claras (Fig. 4). Como exceção, o Caprino 8 evidenciou fígado com coloração vermelha homogênea, mais clara que o normal e sem evidenciação do padrão lobular. Edema na parede da vesícula biliar foi observado nos Caprinos 1 e 6 . Petéquias entre a escápula e o esterno, no epicárdio, mediastino e nas serosas dos órgãos da cavidade abdominal foram observadas em todos os animais. No Caprino 6, o conteúdo do cólon e reto estava ressecado e com muco.

\section{Achados histopatológicos}

Ao exame histológico, as lesões do fígado consistiram de necrose centrolobular com tumefação e discreta vacuolização de hepatócitos periportais (Fig. 5). A lesão foi mais acentuada nos animais que receberam 35 e $40 \mathrm{~g} / \mathrm{kg}$ de peso. Nestes, a necrose, às vezes, atingia quase todo o lóbulo. Nos animais que receberam $30 \mathrm{~g} / \mathrm{kg}$ e folhas verdes à vontade, as áreas de lesões degenerativas foram maiores e a vacuolização de hepatócitos era mais evidente. Os hepatócitos necróticos apresentavam citoplasma eosinofilico com núcleos em picnose ou cariorrexia. Os hepatócitos degenerados tinham citoplasma claro e tumefeito, núcleos ainda íntegros e, ocasionalmente, glóbulos eosinofilicos citoplasmáticos. Hemorragias multifocais de distribuição aleatória associadas à degeneração e necrose hepáticas foram observadas no Caprino 1. As biópsias de fígado efetuadas nos Caprinos 2, 5 e 7 não demonstraram alterações histológicas.

Os encéfalos dos Caprinos 1 e 3 apresentaram tumefação de neurônios, mais proeminente no córtex frontal, associada a edema perineuronal e perivascular. Degeneração hidrópica

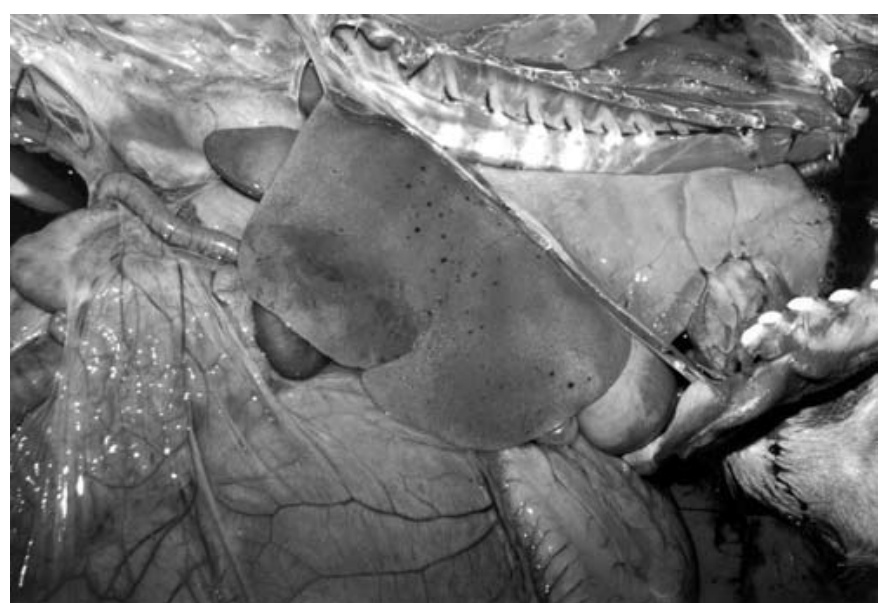

Fig. 3. Intoxicação experimental por Trema micrantha (Caprino 1). Fígado pálido com hemorragias multifocais na superfície capsular.

de grupos de túbulos foi observada no córtex renal dos Caprinos 1 e 8. Os Caprinos 2, 5, 7 e o controle não apresentaram alterações macroscópicas nem histológicas.

\section{DISCUSSÃO E CONCLUSÕES}

Nesse estudo foi caracterizada a toxidez de Trema micrantha, a partir de $30 \mathrm{~g} / \mathrm{kg}$ de peso, em caprinos. Cinco, dos oito caprinos que receberam a planta, morreram. A ingestão voluntária de T. micrantha foi observada no Caprino 6, que consumiu $35,4 \mathrm{~g} / \mathrm{kg}$ de folhas verdes da planta durante o período de 1 dia, sem ter sido submetido a jejum prévio. É provável que todos os animais teriam ingerido as doses propostas de forma espontânea por ela ser palatável.

Os sinais clínicos observados neste experimento são se- 


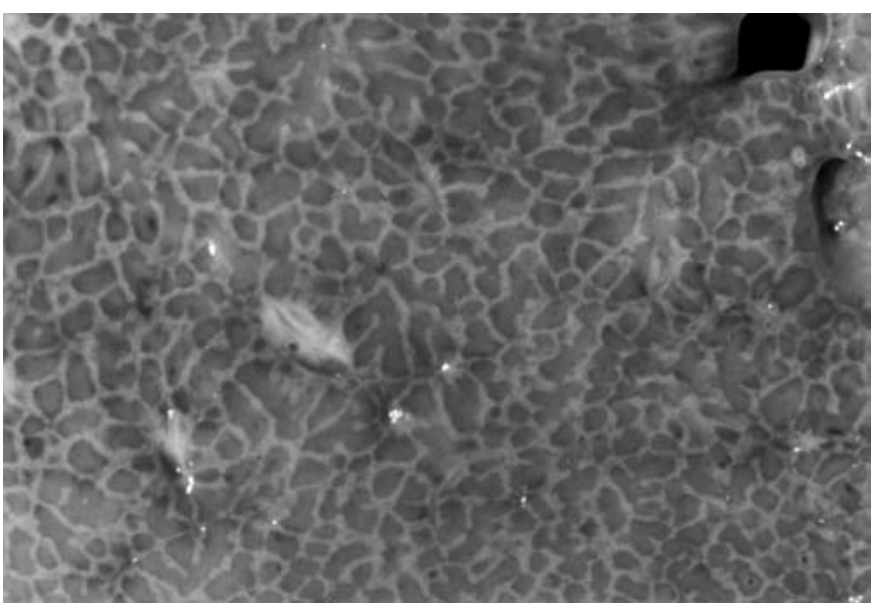

Fig. 4. Intoxicação experimental por Trema micrantha (Caprino 6). Fígado, superfície de corte apresentando lobulação evidente caracterizada por delgadas linhas esbranquiçadas, que correspondiam aos hepatócitos periportais tumefeitos, circundando áreas centrolobulares vermelhas e deprimidas.

melhantes aos relatados nas intoxicações naturais por $T$. tomentosa em camelos (Trueman \& Powel 1991), bovinos, caprinos, ovinos (Mulhearn 1942) e veados (McKenzie 1985). O período necessário para manifestação clínica observada neste estudo foi semelhante ao verificado previamente em experimento com T. tomentosa na mesma espécie (Mulhearn 1942), inclusive, em ambos estudos, utilizaram-se doses muito próximas. Em estudo prévio desenvolvido com coelhos (Traverso \& Driemeier 2000), as alterações clínicas causadas por T. micrantha foram observadas 24 horas antes do que ficou constatado neste experimento, entretanto a dose tóxica observada para coelhos foi $5 \mathrm{~g} / \mathrm{kg}$ maior do que a dos caprinos.

Os sinais clínicos, alterações séricas de GGT, bilirrubina e glicose relatadas neste estudo foram semelhantes aos achados descritos anteriormente envolvendo a intoxicação de caprinos por Vernonia mollissima (Stolf et al. 1987). $O$ aumento significativo de bilirrubina livre no dia da morte dos caprinos, observado na intoxicação por T. micrantha, talvez se deva à lise acentuada de hepatócitos, com consequiente perda na capacidade de conjugação da bilirrubina livre com o ácido glicurônico. Lesões periportais, ainda que moderadas, mas desde que afetem a integridade da membrana celular, resultam em marcada elevação da concentração de pigmentos biliares na circulação (Gopinath \& Ford 1972). Possivelmente, tal aspecto explica o aumento de bilirrubina conjugada que também foi observado no Caprino 1 , no qual houve comprometimento de todo o lóbulo hepático.

Os períodos observados, neste experimento, para ocorrência de manifestação clínica e morte aproximam-se dos descritos na intoxicação por $V$. mollissima em bovinos (Döbereiner et al. 1976). A evolução clínica observada na intoxicação por T. micrantha em caprinos difere daquela encontrada nas intoxicações em bovinos por Sessea brasiliensis (Canella et al. 1968), Cestrum laevigatum (Döbereiner et al. 1969), Cestrum intermedium (Gava et al. 1996) e Xanthium

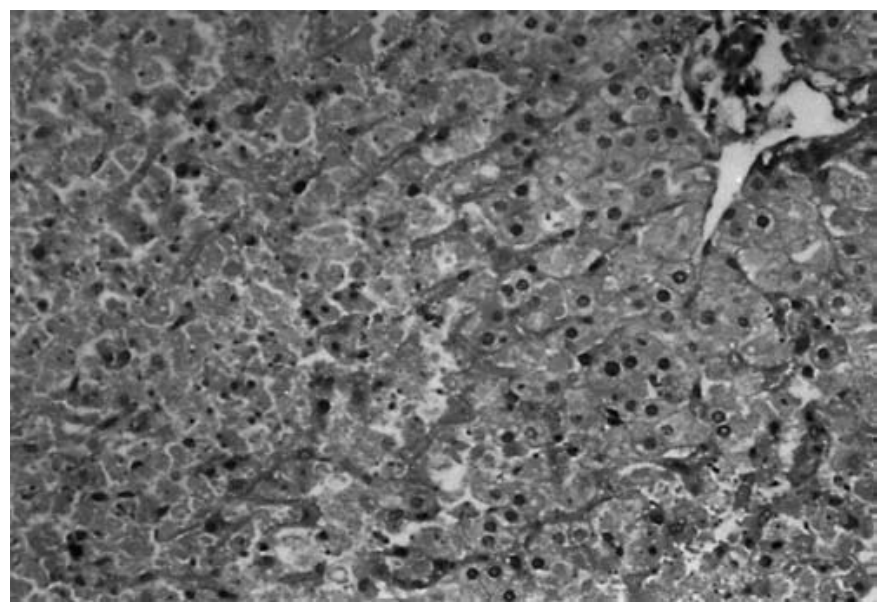

Fig. 5. Intoxicação experimental por Trema micrantha (Caprino 6). Fígado, necrose centrolobular acentuada. HE, obj. 20.

cavanillensii (Driemeier et al. 1999, Colodel et al. 2000), bem como na intoxicação por Vernonia mollissima em caprinos (Stolf et al. 1987), em que as manifestações clínicas ocorreram em períodos não superiores a 36 horas após a ingestão da planta.

Os achados de necropsia descritos na intoxicação experimental por $T$. micrantha são semelhantes aos observados na intoxicação natural descrita em caprinos pela mesma planta (Traverso et al. 2001) e aos constatados nas intoxicações naturais por T. tomentosa em camelos (Trueman \& Powell 1991), bovinos, caprinos, ovinos (Mulhearn 1942), eqüinos (Hill et al. 1985) e veados (McKenzie 1985), bem como aos observados nas intoxicações experimentais por T. tomentosa em bovinos e caprinos (Mulhearn 1942).

As alterações macroscópicas hepáticas aqui descritas são semelhantes às encontradas em intoxicações por Xanthium cavanillesii em bovinos (Driemeier et al. 1999), Cestrum intermedium em bovinos (Gava et al. 1996), C. corymbosum var. hirsutum (Gava et al. 1991), Cestrum parqui em bovinos (RietCorrea et al. 1986), C. laevigatum em caprinos (Peixoto et al. 2000), $V$. mollissima em caprinos (Stolf et al. 1987) e $S$. brasiliensis em bovinos (Canella et al. 1968).

As alterações histológicas observadas no fígado são comuns àquelas descritas nas hepatotoxicoses agudas. Alterações microscópicas no encéfalo foram descritas na intoxicação experimental por T. micrantha em coelhos (Traverso \& Driemeier 2000) e na intoxicação natural por T. micrantha em cabras (Traverso et al. 2002). As lesões encontradas nos coelhos incluíram vacuolização, degeneração e necrose de neurônios corticais, do hipocampo, das células de Purkinje e na substância cinzenta da medula. Em cabras, entretanto, as lesões eram mais proeminentes no córtex frontal e caracterizaram-se por tumefação neuronal e edema perivascular. Lesões encefálicas secundárias à hepatopatia tóxica, descritas na intoxicação por larvas dos dípteros Perreyia flavipes em bovinos e ovinos (Dutra et al. 1997), caracterizaram-se por 
vacuolização da substância branca e, portanto, também diferem daquelas observadas nas intoxicações por T. micrantha. A degeneração hidrópica de túbulos renais, observadas nos Caprinos 1 e 8, não foi descrita anteriormente em casos de intoxicação por plantas hepatotóxicas, embora a degeneração albuminosa granular dos túbulos uriníferos já tenha sido registrada na intoxicação experimental por Vernonia mollissima em caprinos (Stolf et al. 1987).

Os sinais clínicos observados na intoxicação experimental por T. micranta são compatíveis com insuficiência hepática aguda, que se estabelece quando há um comprometimento de $80 \%$ a $90 \%$ do parênquima (Kelly 1993). Muitos fatores, incluindo a hipoglicemia e a falência no mecanismo normal de detoxicação hepática, podem causar aumento excessivo de alguns aminoácidos circulantes, amônia e produtos hepáticos tóxicos, os quais podem induzir alterações neurológicas (Radostits et al. 2000).

A localização centrolobular característica da necrose coagulativa hepatocelular explica-se pela distribuição das enzimas pertencentes ao sistema oxidase microssomal de função mista, que são responsáveis pela transformação de compostos exógenos inativos em metabólitos tóxicos e se encontram em maior concentração nos hepatócitos centrolobulares (Kelly 1993, Maclachlan \& Cullen 1998). Adicionalmente, os hepatócitos da região centrolobular são mais vulneráveis a uma agressão tóxica, por estarem mais distantes do espaço porta, onde o sangue é mais concentrado em oxigênio (Maclachlan \& Cullen 1998).

Edema e hemorragias no sistema nervoso central, bem como alterações neurológicas são achados comuns na insuficiência hepática. $\mathrm{O}$ edema decorre da diminuição na formação de aminoácidos que normalmente são sintetizados no fígado, ocasionando queda nas proteínas plasmáticas e diminuição da pressão oncótica. As hemorragias observadas nesses casos devem-se à deficiência na formação de protrombina e conseqüente aumento no tempo de coagulação. Deficiência de fibrinogênio e tromboplastina também ocorrem (Radostits et al. 2000). A lesão hepática grave causa hiperamonemia, determinando uma alteração histológica no sistema nervoso central que é conhecida como degeneração esponjosa ou status spongiosus. Neste caso, a lesão é caracterizada por vacuolização da substância branca (Hooper 1995). A hipoglicemia tem sido demonstrada como um dos efeitos primários da lesão hepática aguda (Colodel et al. 2000) e esta redução dos níveis sanguíneos de glicose pode ser responsável por sinais de encefalopatia hepática nos casos em que não se verifica hiperamonemia. A lesão histológica observada na hiperamonemia é caracterizada por cromatólise e completo desaparecimento das margens citoplasmáticas, com posterior picnose nuclear (Jubb \& Huxtable 1993). As lesões histológicas do sistema nervoso central, observadas na intoxicação por T. micrantha são restritas à substância cinzenta, sendo mais semelhantes às encontradas na hipoglicemia do que aquelas encontradas na hiperamonemia. Portanto, há possibilidade das lesões neurológicas observadas na presente intoxicação terem sido determinadas pela hipoglicemia, que inclusive foi constatada no Caprino 8 deste estudo. Le- sões no sistema nervoso central também foram observadas na intoxicação por T. micrantha em coelhos (Traverso \& Driemeier 2000).

Os achados macroscópicos e histológicos observados nos animais em experimentação são compatíveis com intoxicação por plantas hepatotóxicas. Os dados epidemiológicos e a presença de $T$. micrantha no local são fundamentais para o diagnóstico da intoxicação natural. Na Região Sul, hepatopatias tóxicas agudas causadas por Xanthium cavanillesii (Driemeier et al. 1999), Cestrum intermedium (Gava et al. 1996), Cestrum corymbosum var. hirsutum (Gava et al. 1991) e Cestrum parqui (Riet-Correa et al. 1986) foram descritas e devem ser levadas em consideração no diagnóstico diferencial. Vernonia mollissima (Stolf et al. 1987) mostrou-se experimentalmente tóxica e Cestrum laevigatum natural e experimentalmente tóxica para caprinos (Peixoto et al. 2000).

Este estudo, além de confirmar a toxidez, de T. micrantha para caprinos, alerta para a possibilidade da ocorrência de casos naturais, cujo diagnóstico pode ser auxiliado pelos dados aqui apresentados. Dentre as plantas comprovadamente hepatotóxicas, T. micrantha é a única que tem sido referida como espécie forrageira (Lorenzi 2000), porém sua utilização em sistemas agroflorestais de silvipecuária deve ser especialmente reconsiderada após as evidências apresentadas no presente estudo. Provavelmente, a intoxicação espontânea não é comum por tratar-se de uma árvore; consequentemente, os animais têm acesso restrito a ela, além de as doses tóxicas serem elevadas.

\section{REFERÊNCIAS}

Barbera R., Trovato A. \& Raspirada A. 1992. Analgesic and anti-inflammatory activity in acute and chronic conditions of Trema micrantha Blume extracts in rodents. Phytotherapy Research 6:146-148.

Braga M.B., Castilhos L.M.L. \& Santos M.N. 1985. Biópsia hepática em bovinos: proposta de nova técnica. Revta Centro Ciênc. Rurais, Santa Maria, 15(1):79-88.

Canella C.F.C., Tokarnia C.H. \& Döbereiner J. 1968. Intoxicação por Sessea brasiliensis Toledo em bovinos. Pesq. Agropec. Bras. 3:333-340.

Castellani E.D. \& Aguiar I.B. 1998. Preliminary conditions for germination of Trema micrantha (L.) Blume seeds. Revista Brasileira de Engenharia Agrícola e Ambiental 2(1):80-83.

Colodel E.M., Driemeier D. \& Pilati C. 2000. Intoxicação experimental pelos frutos de Xanthium cavanillesii (Asteraceae) em bovinos. Pesq. Vet. Bras. 20(1):31-38.

Döbereiner J., Tokarnia C.H. \& Canella C.F.C. 1969. Intoxicação por Cestrum laevigatum Schlecht., a causa da mortandade em bovinos no Estado do Rio de Janeiro. Pesq. Agropec. Bras. 4:165-193.

Döbereiner J., Tokarnia C.H. \& Purisco E. 1976. Vernonia mollissima, planta tóxica responsável por mortandades de bovinos no sul do Mato Grosso. Pesq. Agropec. Bras. 11:49-58.

Driemeier D., Irigoyen L.F., Loretti A.P., Colodel E.M. \& Barros C.S.L 1999. Intoxicação espontânea pelos frutos de Xanthium cavanillesii (Asteraceae) em bovinos no Rio Grande do Sul. Pesq. Vet. Bras. 19(1):1-13.

Dutra F., Riet-Correa F., Méndez M. C. \& Paiva N. 1997. Sawfly (Perreya flavipes) larvae poisoning of cattle and sheep in Uruguay. Vet. Human Toxicol. 39(5):281-286.

Gava A. 2000. Comunicação pessoal (Laboratório de Patologia Animal, UDESC, Lages, SC).

Gava A., Stolf L., Pilati C., Neves D.S. \& Viganó L. 1991. Intoxicação por Cestrum 
corymbosum var. hirsutum (Solanaceae) em bovinos no Estado de Santa Catarina. Pesq. Vet. Bras. 11(3/4):71-74.

Gava A., Stolf L., Varaschin M.S., Neves D.S., Tigre A.P. \& Lessmann F. 1996. Intoxicação por Cestrum intermedium (Solanaceae) em bovinos. Pesq. Vet. Bras. 16(4):117-120.

Gopinath C. \& Ford E.J.H. 1972. Location of the liver injury and extent of bilirubinaemia in experimental liver lesions. Vet. Pathol. 9:99-108.

Hill B.D., Wills L.D. \& Dowling R.M. 1985. Suspected poisoning of horses by Trema aspera (poison peach). Aust. Vet. J. 62(3):107-108.

Hooper P.T. 1975. Spongy degeneration in the central nervous system of domestic animals. Part III. Occurrence and pathogenesis - hepatocerebral disease caused by hyperammonaemia. Acta Neuropathol. 31:343-351.

Jubb K.V.F. \& Huxtable C.R. 1993. The nervous system, p. 267- 440. In: Jubb K.V.F., Kennedy P.C., Palmer N. (ed.) Pathology of Domestic Animals. Vol. 1. $4^{\text {th }}$ ed. Academic Inc., San Diego.

Kelly W.R. 1993. The liver and biliary system, p. 319-406. In: Jubb K. V. F., Kennedy P. C. \& Palmer N. (ed.) Pathology of Domestic Animals. Vol. 2. $4^{\text {th }}$ ed. Academic Inc., San Diego.

Lorenzi H. 2000. Plantas Daninhas do Brasil - terrestres, aquáticas, parasitas e tóxicas $3^{\mathrm{a}}$ ed. Instituto Plantarum de Estudos da Flora, Nova Odessa, SP. 608 p.

Maclachlan N.J. \& Cullen J.M. 1998. Fígado, sistema biliar e pâncreas exócrino, p.1-72. In: Carlton W.W. \& MacGavin M.D. (ed) Patologia Veterinária Especial de Thomson. $2^{\mathrm{a}}$ ed. Artes Médicas, São Paulo.

McKenzie R. A. 1985. Deer farming techniques and diseases of deer in Queensland. Queensland Departament of Primary Industries, Project Report, p. 27.

Méndez M.C., Santos R.C. \& Riet-Correa F. 1998. Intoxication by Xanthium cavanillesii in cattle and sheep in southern Brazil. Vet. Human Toxicol. 40(3):144-147.
Mulhearn C.R. 1942. Poison peach (Trema aspera): a plant poisonous to stock. Aust. Vet. J. 18:68-72.

Nowotny K. \& Nowotny M.P. 1993. Agrossilvicultura baseada na dinâmica e na biodiversidade da Mata Atlântica, p.11-20. In: Jesus E.L., von der Weid J.M., Grzybowski L.M.C. \& Almeida S.G. (ed.) Alternativas - Cadernos de Agroecologia. AS-PTA, Rio de Janeiro. 68 p.

Oelrichs P.B. 1968. Isolation and purification of trematoxin from Trema aspera. Phytochemistry 7:1691-1693.

Peixoto P.V., Brust L.C., Duarte M.D., Franca T.N., Duarte V.C. \& Barros C.S. 2000. Cestrum laevigatum poisoning in goats in southeastern Brazil. Vet. Human Toxicol. 42(1):13-14.

Prophet E.B., Mills B., Arrington J.B. \& Sobin L.H. 1992. Laboratory methods in histotechnology. Armed Forces Institute of Pathology, American Registry of Pathology, Washington. 279 p.

Radostits O.M., Gay C.C., Blood D.C. \& Hinchcliff K.W. 2000. Veterinary Medicine. $9^{\text {th }}$ ed. Baillière Tindall, London. 1763 p.

Riet-Correa F., Schild A.L. \& Méndez M.C. 1986. Intoxicação por Cestrum parqui (Solanaceae) em bovinos no Rio Grande do Sul. Pesq. Vet. Bras. 6(4):111-115.

Stolf L., Gava A. \& Tokarnia C.H. 1987. Intoxicação experimental por Vernonia mollissima (Compositae) em caprinos. Pesq. Vet. Bras. 7(3):67-77.

Tokarnia C.H. \& Döbereiner J. 1982. Intoxicação de bovinos por Vernonia rubricaulis (Compositae) em Mato Grosso. Pesq. Vet. Bras. 2(4):143-147.

Traverso S. \& Driemeier D. 2000. Experimental Trema micrantha (Ulmaceae) poisoning in rabbits. Vet. Human Toxicol. 42(5):301-302.

Traverso S.D., Colodel E.D., Loretti A.P., Correa A.M. \& Driemeier D. 2002. Intoxicação natural por Trema micrantha em caprinos. Ciência Rural, Santa Maria. (Em publicação)

Trueman K.F. \& Powell M.W. 1991. Suspected poisoning of camels by Trema tomentosa (poison peach). Aust. Vet. J. 68(6):213-214. 